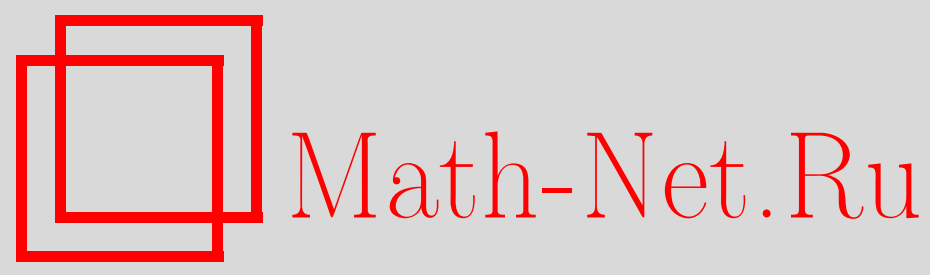

Б. С. Кашин, О возможности обобщения теорем "об исправлении", Матем. заметки, 1997, том 62, выпуск 6, 937-939

DOI: https://doi.org/10.4213/mzm1684

Использование Общероссийского математического портала Math-Net.Ru подразумевает, что вы прочитали и согласны с пользовательским соглашением http://www. mathnet.ru/rus/agreement

Параметры загрузки:

IP: 54.92.164.108

26 апреля 2023 г., 04:19:59

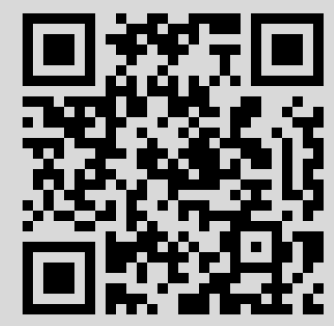




\section{О ВОЗМОЖНОСТИ ОБОБЩЕНИЯ ТЕОРЕМ “ОБ ИСПРАВЛЕНИИ"}

\section{Б. С. Кашин}

В работе автора [1] был установлен аналог известной теоремы Д.Е. Меньшова “об исправлении" для произвольных дискретных полных ортонормированных систем. В [2] при изучении другой задачи теории ортогональных рядов рассмотрены свойства мажоранты частньх сумм ортогонального ряда относительно некоторого семейства подмно-

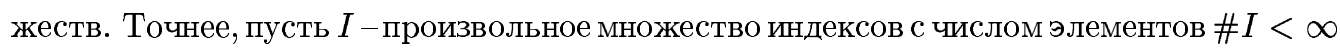
и $\Omega$ - семейство подмножеств $I$. Далее, пусть $\left\{\varphi_{\alpha}\right\}_{\alpha \in I}-$ ортонормированная система функций, заданных на пространстве с мерой $(X, \Sigma, \mu), \mu(X)=1$, и “занумерованных" элементами из $I$. Для ортогонального разложения

$$
f(x) \sim \sum c_{\alpha} \varphi_{\alpha}(x)
$$

определим " $\Omega$-мажоранту частных сумм":

$$
S_{\Omega}^{*} f(x)=\sup _{\Lambda \in \Omega}\left|\sum_{\alpha \in \Lambda} c_{\alpha} \varphi_{\alpha}(x)\right| .
$$

В “обычном" случае $I=I_{N} \equiv\{1,2, \ldots, N\}$ и

$$
\Omega=\left\{I_{k}: 1 \leqslant k \leqslant N\right\} .
$$

Для $d$-кратных ортогональных рядов рассмотрение мажоранты прямоугольных частных сумм сводится к $(1)$, если $I=\left(I_{N}\right)^{d}$, а

$$
\Omega=\left\{I_{k_{1}} \times \cdots \times I_{k_{d}}: 1 \leqslant k_{s} \leqslant N, s=1,2, \ldots, d\right\} .
$$

В этой заметке, дополняющей работу [1], при некоторых условиях на семейство $\Omega$ и систему $\left\{\varphi_{\alpha}\right\}$ установлена возможность исправления произвольной ограниченной функции $f$ до функции с ограниченной $\Omega$-мажорантой. Ниже мы рассматриваем только конечные ортонормированные системы $\Phi_{N}=\left\{\varphi_{k}^{N}(x)\right\}_{k=1}^{N}, N=1,2, \ldots$, удовлетворяющие следуюшим трем условиям:

i) $\left\|\varphi_{k}^{N}(x)\right\|_{L^{\infty}(X, \mu)} \leqslant M_{1}, 1 \leqslant k \leqslant N, N=1,2, \ldots$;

ii) для $N=1,2, \ldots$ существует набор точек $\left\{x_{j}\right\}_{j=1}^{N} \subset X$ такой, что матрица $\left\{N^{-1 / 2} \varphi_{k}^{N}\left(x_{j}\right)\right\}_{k, j=1}^{N}-$ ортонормированная;

iii) для $N=1,2, \ldots$ существует набор точек $\left\{z_{j}\right\}_{j=1}^{Q}$ с $Q \leqslant M_{2} N$ такой, что для любого полинома

$$
P=\sum_{k=1}^{N} a_{k} \varphi_{k}^{N}(x)
$$

имеет место неравенство

$$
\|P\|_{L^{\infty}(X, \mu)} \leqslant M_{3} \max _{1 \leqslant j \leqslant Q}\left|P\left(z_{j}\right)\right|
$$

(здесь и далее $M_{i}, i=1,2, \ldots,-$ абсолютные постоянные).

Работа выполнена при финансовой поддержке Российского фонда фундаментальных исследований, гранты № 96-01-00094 и № 96-15-96102, и фонда INTAS, грант № 93-1376. 
Отметим, что условие іiі) - свойство квазиматричности (или квазидискретности) систем $\Phi_{N}$, введенное в [3], - естественно возникает в ряде вопросов анализа. Кроме того, отметим, что условия і)-іiі) очевидно выполнены в случае, когда при $N=1,2, \ldots$ $\Phi_{N}$ - равномерно ограниченные дискретные системы (т.е. $X=(0,1)$ и $\varphi_{k}^{N}(x)=$ const при $x \in((i-1) / N, 1 / N), 1 \leqslant i \leqslant N)$.

Ниже через $P_{N}\left(x,\left\{y_{k}\right\}_{k=1}^{N}\right)$ мы обозначаем (единственный в силу iі)) полином вида (2), для которого

$$
P\left(x_{k}\right)=y_{k}, \quad 1 \leqslant k \leqslant N .
$$

Введем теперь ограничение на “сложность" последовательности семейств $\Omega_{N}$ подмножеств отрезков натурального ряда $I_{N}, N=1,2, \ldots$ Именно, предположим, что

*) для некоторого $\rho<1$ при $N=1,2, \ldots$ найдутся семейства $\Delta_{s}, \varnothing \in \Delta_{s}, s=$ $1, \ldots, s_{0}, \mathrm{c} \# \Delta_{s} \leqslant M_{4} \exp \exp s^{\rho}$ такие, что каждое множество $\omega \in \Omega_{N}$ допускает представление в виде

$$
\omega=\bigcup_{s=1}^{s_{0}} E_{s}, \quad E_{s} \in \Delta_{s}, \quad \# E_{s} \leqslant \frac{N}{2^{s}}, \quad E_{s} \cap E_{s^{\prime}}=\varnothing \text { при } s \neq s^{\prime} .
$$

ЗАмЕчАниЕ 1 . Близкие к $*$ ) условия на семейства множеств $\Omega_{N}$ играют важную роль в теории коммуникационной сложности (см., например, [4]).

Ниже под исправлением полинома вида (2) мы понимаем замену его на полином $\widetilde{P}$, отличный от $P$ только в малой доле точек $x_{k}, 1 \leqslant k \leqslant N$. Ясно, что для дискретных систем такое исправление есть изменение функции на множестве малой меры.

Метод работы [1] позволяет установить следующий результат

ТЕорема. Пусть при $N=1,2, \ldots$ заданы ортонормированные системы $\Phi_{N}=$ $\left\{\varphi_{k}^{N}(x)\right\}_{k=1}^{N}$, причем выполнены условия i)-іiі), и семейства $\Omega_{N}$ подмножеств $I_{N}$ со свойством *). Для кажсдого $\varepsilon>0$ найдется постоянная $C_{\varepsilon}$, зависящая от $\varepsilon$, а также от $\rho$ и $M_{i}, 1 \leqslant i \leqslant 4$, такая, что при $N=1,2, \ldots$ для любого набора $\left\{y_{j}\right\}_{j=1}^{N} c\left|y_{j}\right| \leqslant 1,1 \leqslant j \leqslant N$, найдется набор $\left\{\widetilde{y}_{j}\right\}_{j=1}^{N}$ такой, что

$$
\#\left\{j: y_{j} \neq \widetilde{y}_{j}\right\} \leqslant \varepsilon N
$$

$u$

$$
\left\|S_{\Omega}^{*} P\left(x,\left\{\widetilde{y}_{j}\right\}\right)\right\|_{L^{\infty}} \leqslant C_{\varepsilon} .
$$

ЗАмЕчАниЕ 2. Метод доказательства теоремы позволяет рассматривать и мажоранты, в определении которых допускается зависимость семейства подмножеств $\Omega$ от точки $x$ (т.е. $\Omega=\Omega(x)$ ).

Как отмечалось в [2], результаты об оценках мажорант $S_{\Omega}^{*}$ применимы к исследованию мажоранты прямоугольных частных сумм кратного ортогонального ряда. В частности, пусть при $d=1,2, \ldots$ и $N=1,2, \ldots$

$$
F_{d, N}=\left\{\bar{\jmath}=\left(j_{1}, \ldots, j_{d}\right) \in \mathbb{Z}^{d}:-N \leqslant j_{s} \leqslant N, s=1, \ldots, d\right\}
$$

и

$$
\Gamma_{d, N}=\left\{z_{\bar{\jmath}}=\left(\frac{2 \pi j_{1}}{2 N+1}, \ldots, \frac{2 \pi j_{d}}{2 N+1}\right), \bar{\jmath}=\left(j_{1}, \ldots, j_{d}\right) \in F_{d, N}\right\} .
$$

Обозначим через $T\left(x,\left\{y_{\bar{\jmath}}\right\}_{\bar{\jmath} \in F_{d, N}}\right)$ тригонометрический полином $t$ степени $\leqslant N$ по каждой из $d$ переменных, для которого $t\left(z_{\bar{\jmath}}\right)=y_{\bar{\jmath}}$, если $z_{\bar{\jmath}} \in \Gamma_{d, N}$. Из теоремы непосредственно вытекает 
СлЕДСТВИЕ. Для $\varepsilon>0 u d=2,3, \ldots$ существует постоянная $C_{\varepsilon, d}$ такая, что для каждого набора $\left\{y_{\bar{\jmath}}\right\} c\left|y_{\bar{\jmath}}\right| \leqslant 1, \bar{\jmath} \in F_{d, N}$, найдется набор $\left\{\widetilde{y}_{\bar{\jmath}}\right\}, \bar{\jmath} \in F_{d, N}, \partial л_{\text {я }}$ которого

$$
\#\left\{\bar{\jmath} \in F_{d, N}: y_{\bar{\jmath}} \neq \widetilde{y}_{\bar{\jmath}}\right\} \leqslant \varepsilon(2 N+1)^{d}
$$

$u$

$$
\max _{\bar{r}}\left\|S_{\bar{r}}\left(T\left(x,\left\{\widetilde{y}_{\bar{j}}\right\}\right)\right)\right\|_{\infty} \leqslant C_{\varepsilon, d}
$$

$2 \partial e$

$$
S_{\bar{r}}(f)=\sum_{\bar{k}:-r_{s} \leqslant k_{s} \leqslant r_{s}} \widehat{f}(k) e^{i \bar{k} x}
$$

- прямоугольная частная сумма тригонометрического ряда Фурье функиии д переменных $f(x)$, а максимум в (3) берется по всем наборам $r=\left\{r_{s}\right\} \in \mathbb{Z}^{d} c 0 \leqslant r_{s} \leqslant N$ nрu $s=1, \ldots, d$.

ЗАмЕчАниЕ 3 . При $d=1$ утверждение следствия было доказано в [5].

Автор признателен А. А. Разборову за полезные обсуждения.

\section{СПИСОК ЦИТИРОВАННОЙ ЛИТЕРАТУРЫ}

1. Кашин Б.С. // Матем. заметки. 1989. Т. 46. №6. С. 67-74. 2. Кашин Б.С., Шарек С.Й. // Матем. заметки. 1995. Т. 58. №2 2. С. 218-230. 3. Кашин Б. С. О тригонометрических полиномах с коэффициентами по модулю равными нулю или единице // Теория функций и приближений. Ч. 1. Саратов: Изд-во Саратовского ун-та, 1987. С. 19-30. 4. Kushilevitz E., Nisan N. Communication Complexity. Cambridge: Cambridge Univ. Press, 1997. 5. Кашин Б. С. // Сооб. АН ГССР. 1979. Т. 93. № 2. C. 281-284. 